\title{
Inkjet Printed Dual Band Antenna for Paper UAVs
}

\author{
S. Jun, J. Heirons and B. Sanz-Izquierdo \\ School of Engineering and Digital Arts, The University of Kent CT2 7NT, Canterbury, Kent, UK \\ b.sanz@kent.ac.uk
}

\begin{abstract}
A dual band antenna is inkjet-printed and then folded as part of a paper unmanned aerial vehicle (UAV). The patterns of the antenna are reproduced on a standard photo paper substrate using an off the shelf inkjet printer. Readily available cartridges with nanoparticle silver conductive ink are employed. A single-layer planar antenna is fed by coplanar waveguide (CPW). The geometry of the radiating element consists of a semicircle with a centered square slot. In order to examine the effect of bending on performance, the antenna is tested unfolded and then folded when integrated onto the airplane. Two configurations of the folded antenna on the plane are analyzed. The aim is to investigate the feasibility of fabricating foldable antennas for paper airplanes using low-cost inkjet printing techniques. The antenna operates at the existing 2.4 GHz and 5.2 GHz WLAN bands. Finite different time domain simulations compare well with measurement.
\end{abstract}

Keywords-Inkjet printing technology; CPW antenna; Origami; Paper; Condutive ink

\section{INTRODUCTION}

In recent years, additive manufacturing (AM) technologies have attracted significant interest in the research and industry communities [1]-[13]. AM have been applied to electronics [1]-[2], mechanical [3]-[4], medical [5]-[7] and microwave and antenna applications [8]-[11]. The development of new materials and inks has been one of the main motivations of this trend. In particular, nanoparticle silver inks have seen a significant uptake [12]-[13]. This inks have been specifically formulated to work with industrial printers and, more recently [13], with standard home inkjet printers. The main advantages of inkjet printing are the speed and accuracy of the fabrication process and the relatively low cost. In the RF and microwave design area, it is desirable to integrate the electronics circuit and devices with the antennas using the flexible materials.

Many different types of antennas fabricated via inkjet printing technology have been reported [14] - [18]. Various substrates have been used, and many applications have been found. A phased-array antenna using inkjet printing technology on flexible Kapton substrate was presented in [14], a monopole antenna for wireless communication and wearable electronics on paper substrate in [15] - [16], a CPW-fed UWB antenna on paper in [17], and on low-cost thermoplastic acrylonitrile butadiene styrene polycarbonate (ABS-PC) substrate in [18]. The latter was demonstrated for integration with UWB sensor networks. Most of these antennas were designed for planar operation.

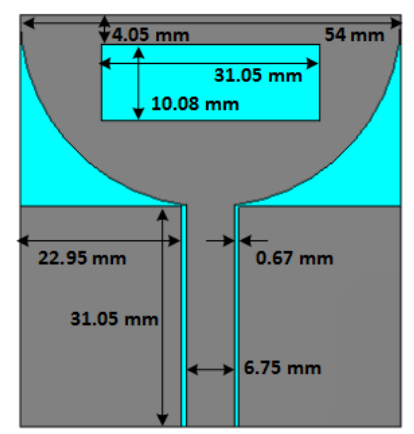

(a)

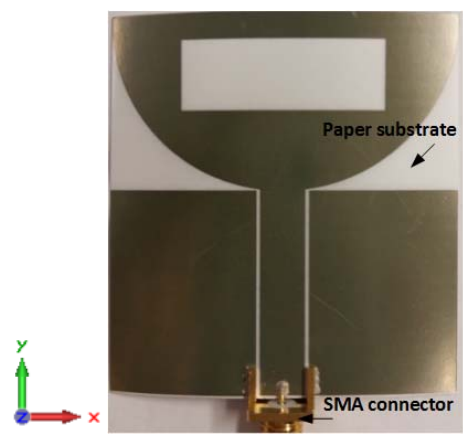

(b)
Fig. 1. Dual band inkjet-printed CPW-fed antenna on paper substrate (a) antenna dimensions, (b) photograph of fabricated antenna with SMA feed connector

This paper presents an inkjet-printed dual-band antenna on paper substrate for origami paper UAVs. The antenna pattern is printed directly onto photo paper using inexpensive inkjet printing technology. The planar antenna is first printed and tested. Then, the antenna is tested when folded on a larger paper sheet, which takes the form of a paper airplane. The antenna is folded on the inside and then to the outside, to investigate the effect of folding on antenna performance and also to find the best condition for communication. The antennas have been designed and optimized, and all the studies have been carried out using the finite difference time domain (FDTD) solver included in CST microwave studio.

\section{ANTENNA DESIGN}

\section{A. Dual band $C P W$-fed antenna}

Fig. 1 shows the geometry of the dual band CPW-fed antenna. The target frequencies of the proposed antenna are $2.4 \mathrm{GHz}$ and $5.2 \mathrm{GHz}$ for the wireless LAN band. The antenna consists of a CPW line and a semicircle with an inner square slot. The semicircle produces the wide bandwidth necessary for the higher frequency bands. The square shifts the lower mode to the $2.4 \mathrm{GHz}$ and filters intermediate bands. The dielectric constant and thickness of the paper used as substrate were set at 3 and $0.177 \pm 12 \mu \mathrm{m} \mathrm{mm}$ respectively. A coaxial cable is used in the simulations in order to replicate the measurement conditions [17]. 


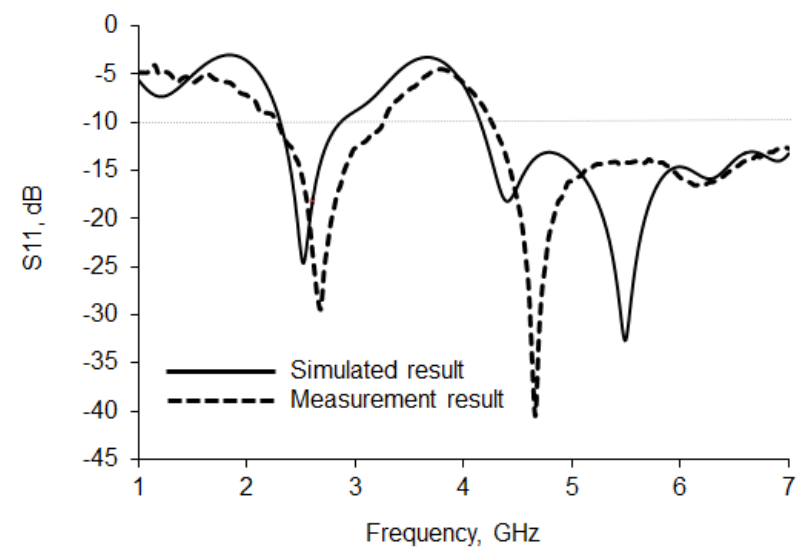

Fig. 2. Simulated and measurement reflection coefficient $\left(\mathrm{S}_{11}\right)$ of the dual band inkjet-printed CPW-fed antenna on paper substrate in free space.

\section{B. Fabrication and measurements}

The model of the antenna was exported from CST microwave studio to ViewMate. It was then converted to a *.gbr file at a 1:1 scale to create a PDF file that could be ready for printing. A Brother MFC-J5910DW inkjet printer was used with AgICCP01A4 paper and AgIC-AN01 Silver Nano Ink [19]. Fig. 1(b) shows the fabricated antenna on the paper substrate. The antenna was fed by a $50 \mathrm{ohm}$ SMA connector which was attached using silver epoxy conductive glue. The antenna was placed in an oven at $70^{\circ} \mathrm{C}$ for an hour to improve the conductivity of the glue and ink. The resistance between the furthest two ends of the antenna was found to be less than $0.5 \mathrm{ohm}$.

Fig. 2 shows the comparison between the simulated and measured reflection coefficients $\left(\mathrm{S}_{11}\right)$ of the antenna. All reflection coefficient measurements were carried using a Rohde \& Schwarz ZVL Vector Network Analyzer. In the simulations, the $-10 \mathrm{~dB}$ bandwidth spans from 2.3 to $2.8 \mathrm{GHz}$, and from 4.1 to over $7 \mathrm{GHz}$ at the 2.4 and $5.2 \mathrm{GHz}$ respectively. In the measurement, the resonant frequencies shifted to the right. The $-10 \mathrm{~dB}$ bandwidth spans from 2.3 to 3.2 $\mathrm{GHz}$ at the lower band, and from 4.2 to over $7 \mathrm{GHz}$ at the higher band. The difference between the computed and measured results is believed to be due to the SMA connector and feeding cables.

\section{FOLDED ANTENNA ON PAPER AIRPLANE}

\section{A. Intial Desgin of Folded antenna design}

The substrate of the designed dual band CPW-fed antenna was extended to enable folding to the shape of a paper airplane. The overall area was $210 \mathrm{~mm} \times 282.5 \mathrm{~mm}$. The dimensions of the antenna were as described in section II (Fig.1 (a)). Two cases were first investigated and are shown in Fig. 3. The first (Fig.3a) is the planar antenna in Fig.1 with the paper substrate extended. The second (Fig.3b) is the same antenna folded on the inside as part for the origami paper airplane.

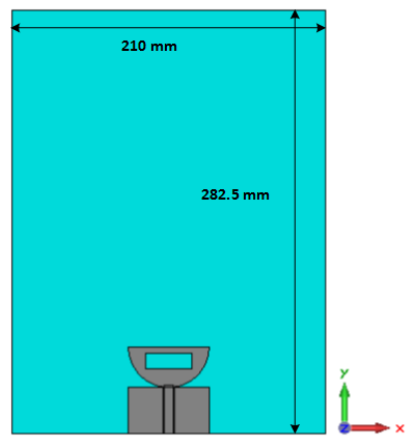

(a)

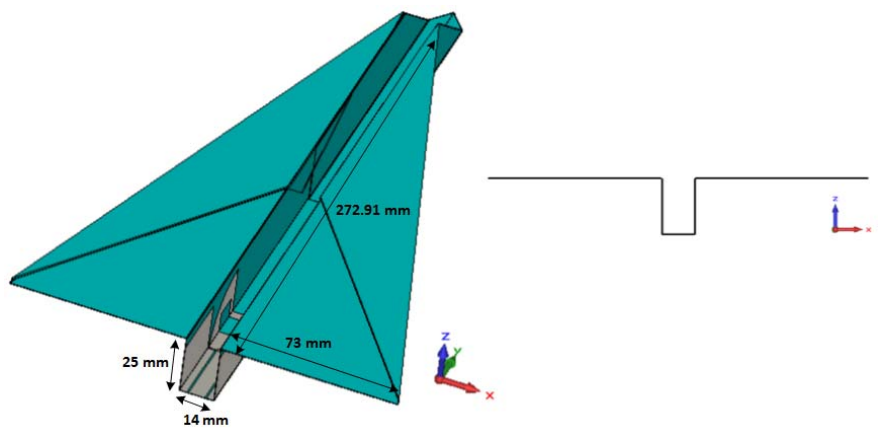

(b)

Fig.3. Dual band inkjet-printed CPW-fed antenna on the paper substrate: (a) extended planar, (b) folded type 1

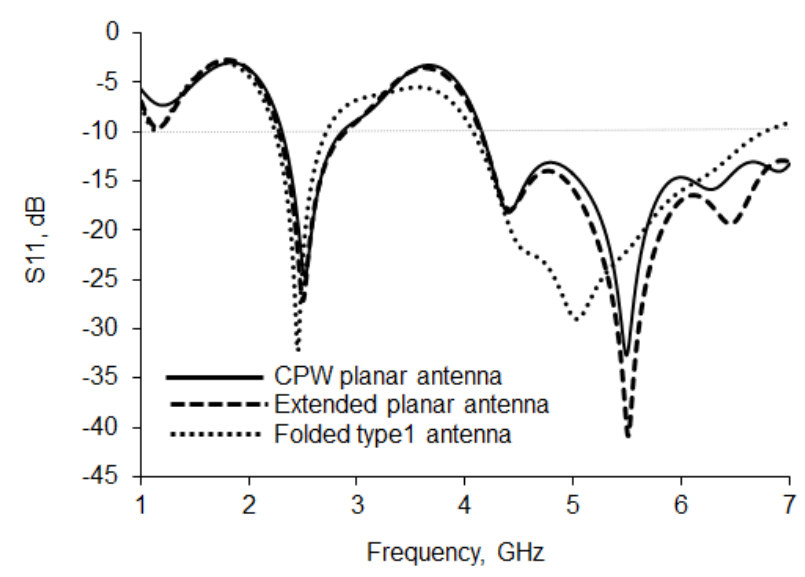

Fig. 4. Computed $\mathrm{S}_{11}$ of the dual band inkjet-printed CPW-fed antenna on the paper substrate

Fig. 4 presents the reflection coefficient $\left(S_{11}\right)$ for the three cases: the planar antenna (Fig.1a), the planar antenna with extended paper sheet (Fig.3a), and the folded antenna (Fig.3b). The overall increase in dimensions of the paper substrate had almost no influence on the antenna performance. When the antenna is folded, the resonant frequency shifted very slightly to the left, and bandwidth also decreased. The matching of the antenna $\left(S_{11}\right)$ improved at the lower band but degraded slightly at the higher band. 


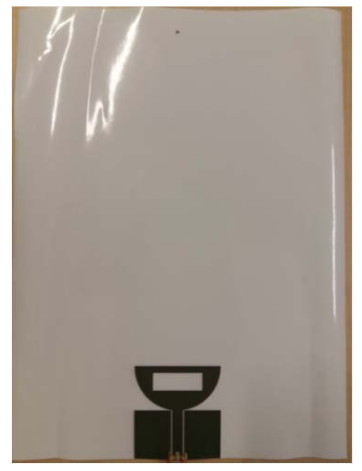

(a)

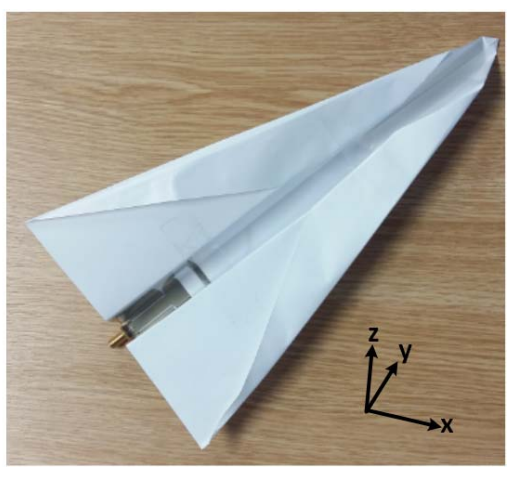

(b)
Fig. 5. Photographs of (a) the planar antenna on the extend paper substrate, and (b) the folded type 1 antenna

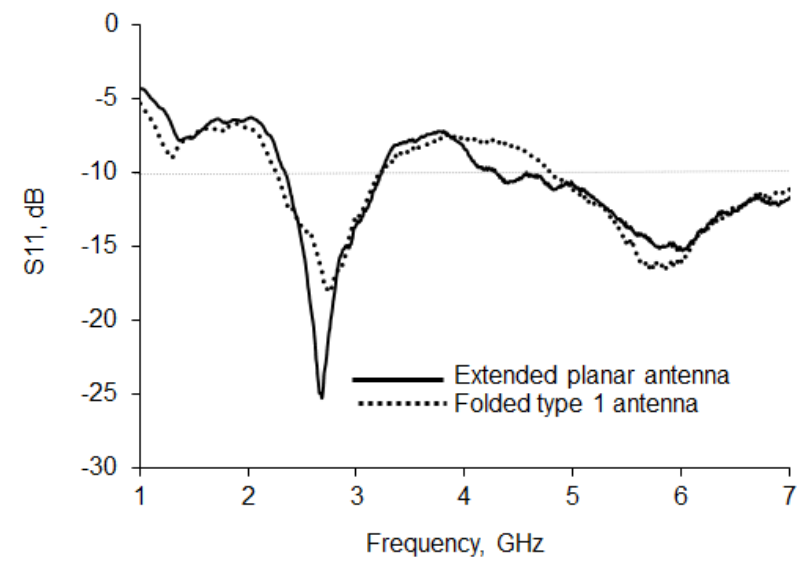

Fig. 6. Measured $\mathrm{S}_{11}$ of the extended planar, and folded antennas on the paper substrate

\section{B. Fabrication and mesurement}

The antennas were fabricated using the same procedure described in section II B. A very simple and traditional origami folding airplane was used for this first demonstrator. The unfolded and the initial folded antennas fabricated are shown in Fig.5. The measured $S_{11}$ of the two antennas is presented in Fig. 6. As with the simulation, the measured reflection coefficients of the folded antennas at lower frequency is worse than for the planar antennas. Nevertheless, the two designs were able to cover the 2.4 and $5.2 \mathrm{GHz}$ WLAN bands with an $\mathrm{S}_{11}$ level of at least $-10 \mathrm{~dB}$.

The simulated radiation patterns at 2.4 and $5.2 \mathrm{GHz}$ are shown in Fig. 7 and 8 respectively. The simulated radiation patterns of the folded antennas are similar to the planar antenna except in the positive $\mathrm{z}$ direction. The antenna becomes more directional when folded, particularly at the higher band. The peak gain of 3.74 and $4.96 \mathrm{~dB}$ are obtained at 2.4 and $5.2 \mathrm{GHz}$ respectively.

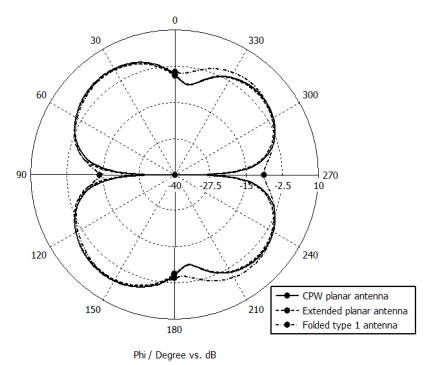

(a)

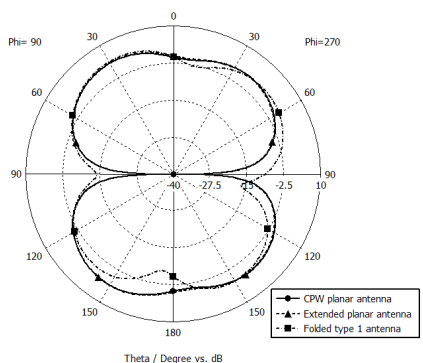

(b)

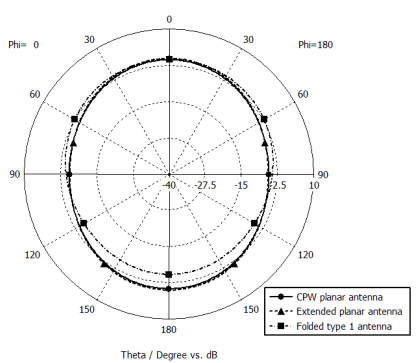

(c)

Fig. 7. Radiation patterns at 2.4 GHz (a) XY plane (b) YZ plane (c) XZ plane

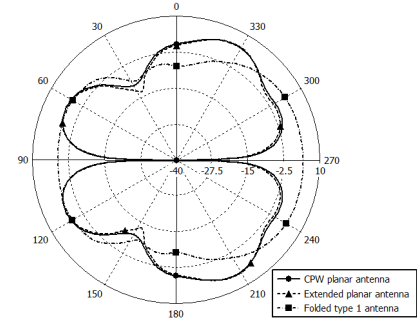

(a)

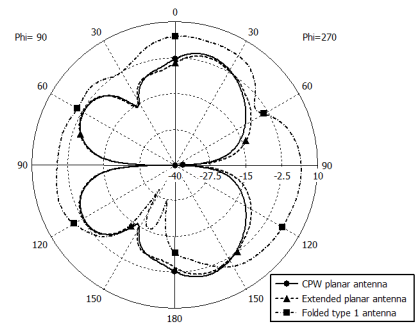

(b)

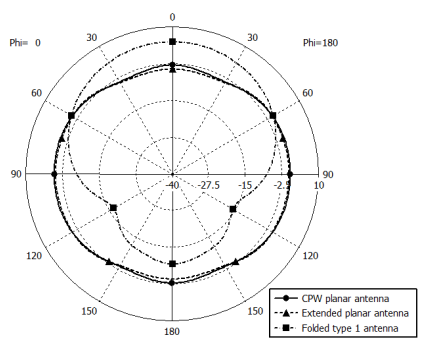

(c)

Fig. 8. Radiation patterns at 5.2 GHz (a) XY plane (b) YZ plane (c) XZ plane

\section{Towards an optimised origami plane antenna design}

Ideally, the antenna on the paper UAV should transmit efficiently and the radiation pattern should point towards the remote controller. The initial folded antenna design (Type 1) had a radiation pattern which pointed slightly upwards (Fig.7, Fig. 8). This may not be the best solution when the controller is positioned at lower height than the airplane. This section describes a potential technique for modifying the pattern of the antenna. 


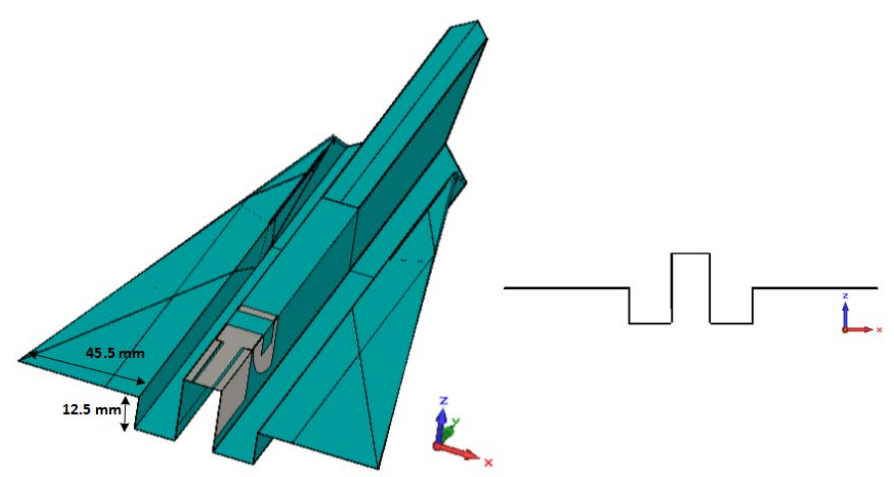

Fig. 9. Folded dual band inkjet-printed CPW-fed antenna (type 2)

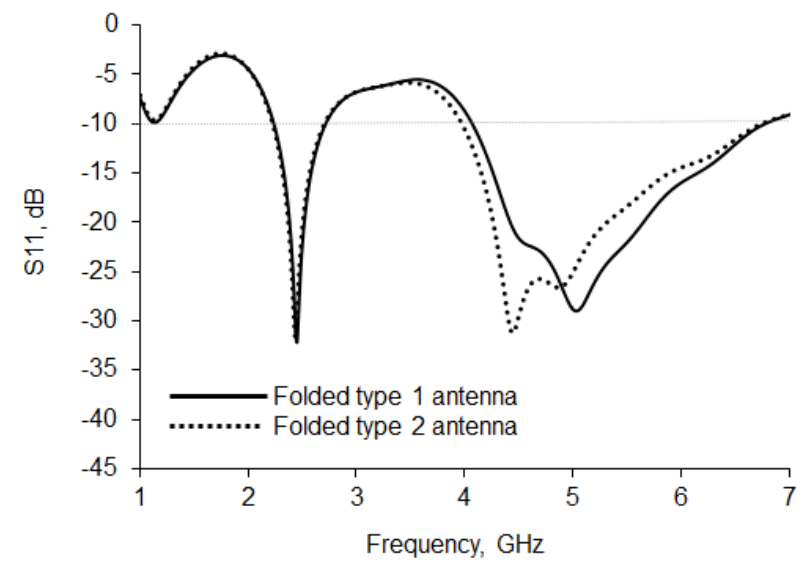

Fig. 10. Simulated $S_{11}$ of the folded dual band inkjet-printed CPW-fed antennas

Fig. 9 shows the same antenna with a new folding method for the origami airplane. The new folded antenna gave very similar $S_{11}$ (Fig.10) to the first folded design ((Fig.3 (b)). Fig. 11 shows the fabrication prototype while Fig. 12 presents the measured $\mathrm{S}_{11}$. The higher band is clearly more sensitive to folding than the lower band. The new design was able to cover the desired 2.4 and $5.2 \mathrm{GHz}$ WLAN bands. Moreover, the radiation pattern (Fig.13 and 14) is pointing downwards which could prove to be more efficient in the case of the controller being at a lower height than the UAVs.

\section{CONCLUSIION AND DiscusSION}

The inkjet printing and folding of a WLAN antenna for paper airplanes has been demonstrated. A commercially available, low-cost, inkjet printer with cartridges filled with nanoparticle silver ink is able to produce functional antennas for this application. The paper-based antenna has acceptable performance in planar and folding conditions. The folding should be very smooth as there is a potential for cracking the silver conductive traces. Folding the antenna increases its directivity. This may steer the beam towards an unwanted direction. By changing the folding technique and the design of the airplane, it is possible to optimize the direction of the radiation pattern.

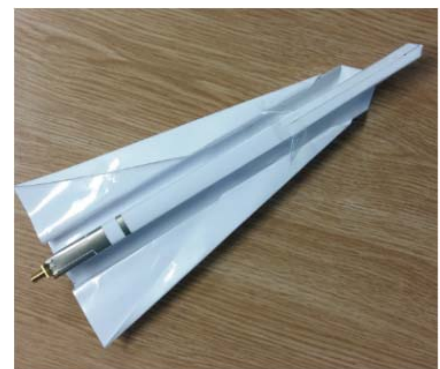

Fig. 11. Photographs of the inkjet-printed and folded CPW-fed antenna (type 2)

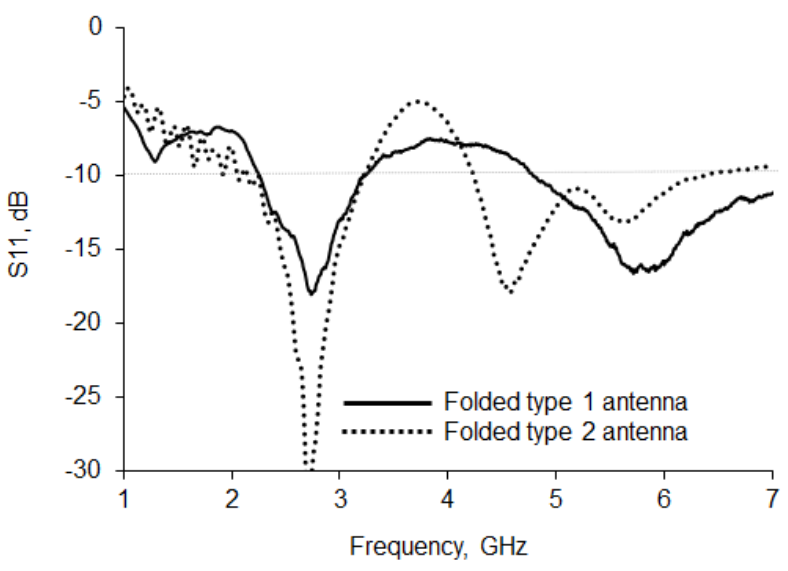

(b)

Fig. 12. Measured $\mathrm{S}_{11}$ of the folded dual band inkjet-printed CPW-fed antennas

This paper has provided a basic design concept for foldable antenna on UAVs. Ongoing work is trying to optimize the antenna for different remote controller locations and also apply new origami folding techniques for paper UAVs.

\section{ACKNOWLEDGMENT}

The authors would like to thank Simon Jakes for help with fabrication, and Cyril Isenberg for helpful discussions. This work was funded by the UK Royal Society and the UK EPSRC High Value Catapult Fellowship.

\section{REFERENCES}

[1] E. Macdonald, R. Salas, D. Espalin, M. Perez, E. Aguilera, D. Muse and R. Wicker, "3D Printing for the Rapid Prototyping of Structural Electronics", IEEE Access, vol. 2, pp. 234-242, 2014.

[2] J. Hester, S. Kim, J. Bito, T. Le, J. Kimionis, D. Revier, C. Saintsing, W. Su, B. Tehrani, A. Traille, B. Cook and M. Tentzeris, "Additively Manufactured Nanotechnology and Origami-Enabled Flexible Microwave Electronics", Proceedings of the IEEE, vol. 103, no. 4, pp. 583-606, 2015.

[3] A. Griffiths, A. Dikarev, P. Green, B. Lennox, X. Poteau and S. Watson, "AVEXIS-Aqua Vehicle Explorer for In-Situ Sensing", IEEE Robot. Autom. Lett., vol. 1, no. 1, pp. 282-287, 2016.

[4] Y. He, S. Guo, L. Shi, S. Pan, and Z. Wang, "3D printing technologybased an amphibious spherical robot," in Proc. IEEE Int. Conf. Mechatron. Autom. (ICMA'14), pp. 1382-1387, Aug. 3-6, 2014

[5] F. Rengier, A. Mehndiratta, H. von Tengg-Kobligk, C. Zechmann, R. Unterhinninghofen, H. Kauczor and F. Giesel, "3D printing based on 
imaging data: review of medical applications", Int J CARS, vol. 5, no. 4, pp. 335-341, 2010.

[6] A. Cloonan, D. Shahmirzadi, R. Li, B. Doyle, E. Konofagou and T. McGloughlin, "3D-Printed Tissue-Mimicking Phantoms for Medical Imaging and Computational Validation Applications", $3 D$ Printing and Additive Manufacturing, vol. 1, no. 1, pp. 14-23, 2014.

[7] J. Scoggin and T. A. Murray, "Novel Uses of 3D Printing for in vitro Biomedical Research," 2016 32nd Southern Biomedical Engineering Conference (SBEC), Shreveport, LA, pp. 29-30, 2016.

[8] J. C. Batchelor, E. A. Parker, J. A. Miller, V. Sanchez-Romaguera, and S. G. Yeates, "Ink jet printing of frequency selective surfaces," Electron. Lett., vol. 45, no. 1, pp. 7-8, Jan. 2009.

[9] B. Sanz-Izquierdo and E. A. Parker, "3D printing of elements in frequency selective arrays," IEEE Trans. Antennas Propag., vol. 62, no. 12, pp. 6060-6066, Dec. 2014.

[10] S. Jun, B. Sanz-Izquierdo and E. A. Parker, "3D printing technique for the development of non-planar electromagnetic bandgap structures for antenna applications," Electron. Lett., vol. 52, no. 3, pp. 175-176, Feb. 2016.

[11] S. Jun, B. Sanz-Izquierdo, and M. Summerfield, "UWB antenna on 3D printed flexible substrate and foot phantom," in Antennas \& Propagation Conference (LAPC), 2015 Loughborough, vol., no., pp.1-5, 2-3 Nov. 2015.

[12] S. Khan, R. Dahiya, and L. Lorenzelli, "Technologies for printing sensors and electronics over large flexible substrates: A review," IEEE Sensors J., vol. 15, no. 6, pp. 3164-3185, Jun. 2015.

[13] Y. Kawahara et al., "Instant Inkjet Circuits: Lab-Based Inkjet Printing to Support Rapid Prototyping of UbiComp Devices," Proc. 2013 ACM Int'l Joint Conf. Ubiquitous Computing (UbiComp 13), pp. 363-372, 2013.

[14] H. Subbaraman, D. T. Pham, X. C. Xu, M. Y. H. Chen, A. Hosseini, X. $\mathrm{Lu}$, and R. T. Chen, "Inkjet-Printed Two-Dimensional Phased-Array Antenna on a Flexible Substrate," IEEE Antennas Wireless Propag. Lett., vol. 12, pp. 170-173, 2013

[15] H. F. Abutarboush, A. Shamim, "Paper-Based Inkjet-Printed Tri-Band U-Slot Monopole Antenna for Wireless Applications," IEEE Antennas Wireless Propag. Lett., vol.11, pp.1234-1237, 2012.

[16] S. Kim, Y. J. Ren, H. Lee, A. Rida, S. Nikolaou, M. M. Tentzeris, "Monopole Antenna With Inkjet-Printed EBG Array on Paper Substrate for Wearable Applications", IEEE Antennas Wireless Propag. Lett., vol. 11, pp: $663-666,2012$.

[17] G. Shaker, S. Safavi-Naeini, N. Sangary, and M. Tentzeris, "Inkjet printing of ultrawideband (UWB) antennas on paper-based substrates," IEEE Antennas Wireless Propag. Lett., vol. 10, pp. 111-114, 2011.

[18] D. Unnikrishnan, D. Kaddour, S. Tedjini, E. Bihar, M. Saadaoui, "CPW-Fed Inkjet Printed UWB Antenna on ABS-PC for Integration in Molded Interconnect Devices Technology," IEEE Antennas Wireless Propag. Lett., vol.14, no., pp.1125-1128, 2015.

[19] [online] https://agic.cc/en/ (Accessed 06/10/2016)

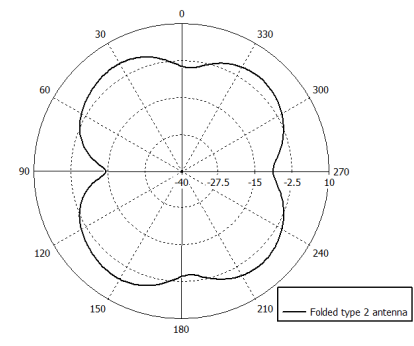

(a)

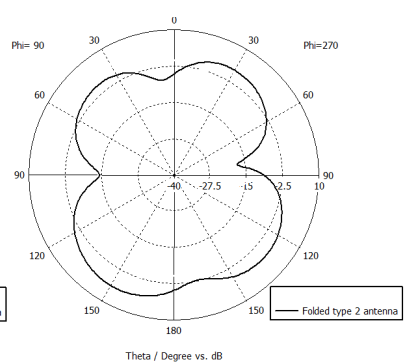

(b)

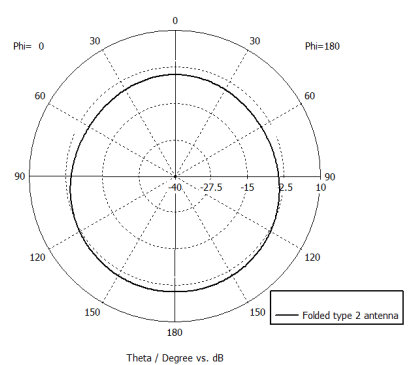

(c)

Fig. 13. Radiation patterns at 2.4 GHz (a) XY plane (b) YZ plane (c) XZ plane

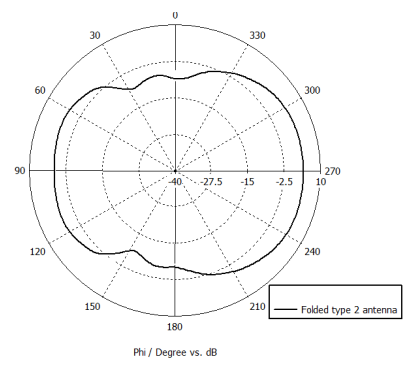

(a)

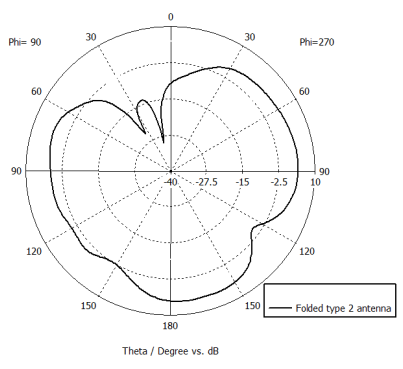

(b)

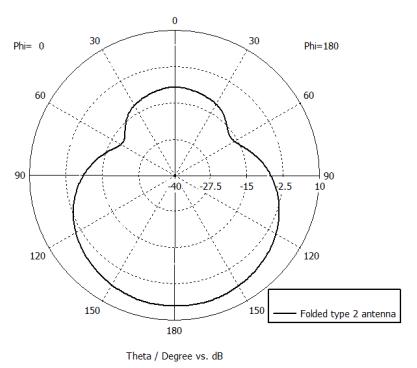

(c)

Fig. 14. Radiation patterns at 5.2 GHz (a) XY plane (b) YZ plane (c) XZ plane 